Proceedings

\title{
Degradation and Protection of Entanglement in Open Quantum Systems ${ }^{\dagger}$
}

\author{
Antonella De Pasquale ${ }^{1,2, \ddagger}$ \\ 1 Department of Physics and Astronomy, University of Florence, 50019 Sesto Fiorentino (FI), Italy; \\ adepasquale83@gmail.com \\ 2 NEST, Scuola Normale Superiore and Istituto Nanoscienze-CNR, I-56127 Pisa, Italy \\ + Presented at the 11th Italian Quantum Information Science Conference (IQIS2018), Catania, Italy, \\ 17-20 September 2018. \\ $\ddagger$ Current address: Via G. Sansone 1, 50019 Sesto Fiorentino (FI), Italy.
}

Published: 23 July 2019

check for updates

\begin{abstract}
The distribution of entangled quantum systems among the nodes of a network is a key task at the basis of the development of quantum technologies, e.g., quantum communication, quantum computation, etc. Many efforts have been devoted to identify strategies, based on pre- and post-processing operations or decoherence-free subspaces, to prevent the deterioration of such exotic correlations. However, all these approaches loose their usefulness when the noise level affecting the system surpasses a certain minimal threshold that leads to an entanglement-breaking dynamics. Here we attack this problem in the context of discrete- and continuous-time description of the system dynamics, providing some explicit examples in the context of qubit channels.
\end{abstract}

Keywords: entanglement-breaking channels; Markovian dynamics; non-Markovian dynamics

\section{Introduction}

Real quantum transmission lines are typically affected by external perturbations due to spoil interactions with the external environment, inducing a gradual degradation of the transmitted signals. From a mathematical point of view the evolution of a quantum system, either in time or in space, is described by a quantum channel [1], a superoperator transforming the input into the output state. Is it possible to characterize the quality of such evolution? A possible criterion to draw a sort of noise spectrum for the dynamical evolution of a physical system consists in quantifying the portion of the entanglement initially shared between the evolving system and an arbitrary ancilla that survives at the end of transmission process [2]. The ideal case, characterized by the absence of losses, is represented by unitary maps, suited for the description of closed systems undergoing coherent evolution. More in general, dynamical maps are not perfect and a fraction of the initial correlations can be lost. In order to cope with this effect many error-correction protocols, based on pre- and post-processing operations, have been designed so as allow for an efficient entanglement distribution through imperfect channels. Such strategies are however completely ineffective when the system evolution is described by an entanglement-breaking (EB) channel [3]: these maps are so leaky and noisy that any initial state of the system and the ancilla will end up to become separable. In such a case any encoding and decoding operation, acting respectively on the input and output states of the map, results completely useless since, once destroyed, entanglement cannot be generated by local operations or classical communications. The only possibility to overcome this hindrance consists in manipulating the transmission line itself. The aim of this work is discussing some techniques in this direction. We will also provide some examples dealing with qubit systems, in some cases are also corroborated by experimental evidence. 


\section{Materials and Methods}

The evolution of a quantum system $S$ is in general described by a linear, completely positive, trace-preserving $(\mathrm{CPt})$ mapping $\Phi$, the quantum channel, which associates an output state $\Phi\left(\rho_{S}\right)$ to each possible initial density matrix $\rho_{S}$ of $S$. Two channels $\Phi$ and $\Psi$ can be combined yielding a third map $\Phi \circ \Psi$ such that $\Phi \circ \Psi\left(\rho_{S}\right)=\Phi\left(\Psi\left(\rho_{S}\right)\right)$. A quantum channel said to be EB if, for all its extensions to an ancillary system $A$, it annihilates the entanglement between the system and the ancilla, i.e., if $\Phi \otimes \operatorname{Id}\left(\rho_{S A}\right)$ is separable for all input states $\rho_{S A}$ of the composite system $S A$, Id being the identity map acting on the ancilla. Notice that the set of EB maps is stable under concatenation with other (not necessarily entanglement-breaking) maps, i.e if $\Phi$ is EB we have that the maps $\Phi \circ \Psi$ and $\Psi \circ \Phi$ are EB for any channel $\Psi$. Moreover, for finite dimentional systems, on which we will focus in this manuscript, the Choi-Jamiołkowski isomorphism allows to restrict the analysis to the case where the ancilla $A$ is isomorphic to $S$ and $\rho_{S A}$ is the maximally entangled state $|\Omega\rangle_{A S}=\frac{1}{\sqrt{d}} \sum_{k=1}^{d}|k\rangle_{A} \otimes|k\rangle_{S}$, $\{|k\rangle\}_{k=1, \cdots, d}$ being an orthonormal basis on the associated Hilbert space. The output density matrix $\Phi \otimes \operatorname{Id}\left(|\Omega\rangle_{A S}\langle\Omega|\right)$ is called the Choi-Jamiołkowski state of $\Phi$, and its separability is equivalent to the EB property of the map.

If we are interested in a description of the system evolution such that the time $t$ explicitly appears as a continuous parameter, we can resort to a family of maps $\left\{\Phi_{t, 0}\right\}_{t \geq 0}$, where $t=0$ indicates the initial time. In general there is a two-way flow of information from the system to the environment and viceversa. If the information back-flow from the environment to the system can be discarded, we say that the evolution is Markovian [4], which from a mathematical point of view amounts to say that all the elements of the set $\left\{\Phi_{t, 0}\right\}_{t \geq 0}$ satisfy the CP-divisibility property, $\Phi_{t, 0}=\Lambda_{t, t^{\prime}} \circ \Phi_{t^{\prime}, 0}, \forall t \geq t^{\prime} \geq 0$, being $\Lambda_{t, t^{\prime}}$ a completely-positive map. The stability under concatenation of the set of EB maps and the $\mathrm{CP}$-divisibility property held by Markovian dynamics imply that once the entanglement between the evolving system $S$ and any other ancilla $A$ is lost, it cannot be recovered anymore. In this respect we can characterize the noise level of the evolution from the point of view of entanglement transmission by computing the smallest time $\tau_{\text {ent }}$ for which $\Phi_{t, 0}$ becomes EB. This quantity has been introduced and studied in [5] under the name of entanglement survival time. An important subclass of Markovian (CP-divisible) processes is provided by the so-called dynamical semi-groups, characterized by channels which are invariant under translations of the time coordinates, that is such that $\Lambda_{t, t^{\prime}}=\Lambda_{t-t^{\prime}, 0}=\Phi_{t-t^{\prime}, 0}$, $\forall t \geq t^{\prime}$. This condition enables to write the so-called Gorini-Kossakowski-Sudarshan-Lindblad (GKSL) master equation for the dynamical evolution of the system [6,7]

$$
\frac{\partial \rho_{S}(t)}{\partial t}=\mathcal{L}\left[\rho_{S}(t)\right]=-i \omega\left[H, \rho_{S}(t)\right]+\gamma \mathcal{D}\left[\rho_{S}(t)\right]
$$

where $\mathcal{L}$ is the Lindblad generator of the dynamics, characterized by two main contributions: a unitary Hamiltonian term proportional to $\omega$ and a purely dissipative term $\mathcal{D}$ governing the irreversible process. The frequencies $\omega$ and $\gamma$ in (1) gauge the relative strengths of these two terms. It can be easily proved that $\tau_{\text {ent }}$ depends on the ratio $k=\omega / \gamma$, from which one can introduce the dimensionless quantity $\mathcal{T}_{\text {ent }}(k)=\gamma \tau_{\text {ent }}$. Intuitively, one would aspect that a larger incidence of the unitary driving with respect to the purely dissipative contribution would yield longer values of $\tau_{\text {ent }}$. Actually this is not always the case, and in some circumstances the presence of a non-zero value of the Hamiltonian parameter $\omega$ induces a drastic reduction of the entanglement survival time, see Figure 1.

While justified in certain contexts, the Markov approximation fails when the system-environment interaction leads to long-lasting and non-negligible correlations. In general, the continuous time dynamics of an open system is described by an integro-differential equation, accounting for the fact that the future dynamics of the system depends on all its past history. From the point of view of correlations transmission, this may lead to the reappearance of entanglement after its disappearance, a criterion actually proposed as a non-Markovianity witness [8]. 


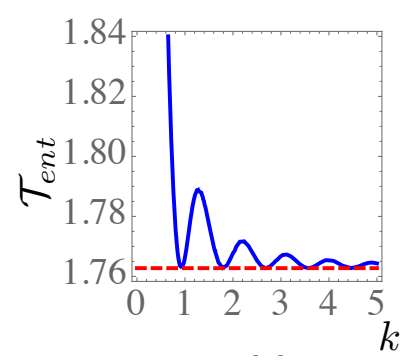

Figure 1. Study of the rescaled entanglement survival time $\mathcal{T}_{\text {ent }}(k)$ for the phase-flip process for qubit systems, described by the Lindblad generator $\mathcal{L}[\ldots]=\frac{\gamma}{2}\left(\sigma_{z}[\ldots] \sigma_{z}-\mathbb{I}\right)-i \omega\left[\sigma_{x}, \ldots\right]$. On average it results a tendency of the model in translating high level of unitary driving into a stronger entanglement suppression.

\section{Results}

An EB channel can be visualized as a communication line able to transmit entanglement only up to a fixed length. It has been proved [4,9-11] that it is possible to restore the transferring of quantum correlations via proper manipulations of the map itself. In Figure 2a we show that if an EB map $\mathcal{M}$ is divisible in smaller pieces $\Phi$ of equal length, i.e., $\mathcal{M}=\Phi \circ \Phi$, one can possibly restore entanglement transmission by inserting a proper (unitary) filter $\mathcal{F}$ between the subchannels. In Figure $2 \mathrm{~b}$ instead, we assume to start with two EB maps decomposable as $\mathcal{M}_{1}=\Phi \circ \Phi$ and $\mathcal{M}_{2}=\Psi \circ \Psi$, and show that by reshuffling their subcomponents it is possible to build up a new communication line able to transmit entanglement. The proof-of-principle demonstration for these effects has been realized for qubit systems, by choosing $\Phi$ and $\Psi$ as given by rotated amplitude damping channels, $\Phi=\mathcal{A}_{\eta} \circ \mathcal{U}_{\theta}$ and $\Psi=\mathcal{U}_{\varphi} \circ \mathcal{A}_{\eta}$. The amplitude damping channel $\mathcal{A}_{\eta}$ accounts for energy dissipation processes and transforms an input density matrix as $\mathcal{A}_{\eta}(\rho) \equiv E_{1} \rho E_{1}^{+}+E_{2} \rho E_{2}^{+}$, where $E_{1} \equiv\left[\begin{array}{cc}1 & 0 \\ 0 & \sqrt{\eta}\end{array}\right]$ and $E_{2} \equiv\left[\begin{array}{cc}0 & \sqrt{1-\eta} \\ 0 & 0\end{array}\right]$. The unitary operation $\mathcal{U}_{\theta}$, playing also the role of the filter $\mathcal{F}$ in Figure $2 \mathrm{a}$, is given by $\mathcal{U}_{\varphi}(\rho)=R_{\varphi} \rho R_{\varphi}$, with $R_{\varphi} \equiv\left[\begin{array}{cc}\cos (2 \varphi) & -\sin (2 \varphi) \\ -\sin (2 \varphi) & \cos (2 \varphi)\end{array}\right]$. The presented examples have been also tested in quantum optics experiments based on the transmission of single-photon polarization states $[9,10]$. Such techniques have been proved to work also for continuous-variable quantum systems [11,12] and for the case of continuous-time Markovian evolution [9].

In a non-Markovian setting the concurrence can exhibit a nondecreasing behavior due to information back-flow from the environment. Therefore entanglement can eventually reach zero at the special times (or time intervals) where the process becomes EB, and then reappear again (see the solid blue line in Figure 2c). In [13] it has been shown that the EB properties of a non-Markovian evolution can be manipulated by properly acting on the environment via periodic resetting of its initial state. This is formally realized by dividing the temporal axis into a collection of time intervals $I_{n}=\left[t_{n}, t_{n+1}\right)$ of equal length $\tau=t_{n+1}-t_{n}$ with $t_{0}=0$, and by defining a new family of mappings $\left\{\tilde{\Phi}_{0, t}^{(\tau)}\right\}_{t \geq 0}$ as $\tilde{\Phi}_{0, t}^{(\tau)}=\Phi_{0, t-n \tau} \circ\left(\Phi_{0, \tau}\right)^{n}$ for $t \in \mathcal{I}_{n}$. In Figure 2c we report a case study regarding a qubit $S$ interacting with a bosonic reservoir at zero temperature characterized by a Lorentzian spectral density. It has been observed that for sufficiently small $\tau$ the concurrence of the Choi-Jamiołkowski state never vanishes for all $t$, reaching the constant value of 1 as $\tau$ approaches zero. This can be accounted to the fact that in this limit the dynamics described by $\tilde{\Phi}_{0, t}^{(\tau)}$ gets effectively frozen, thus establishing an interesting connection between this protocol and the quantum Zeno effect, which takes place whenever a strong disturbance dominates the time evolution of a quantum system [14]. 


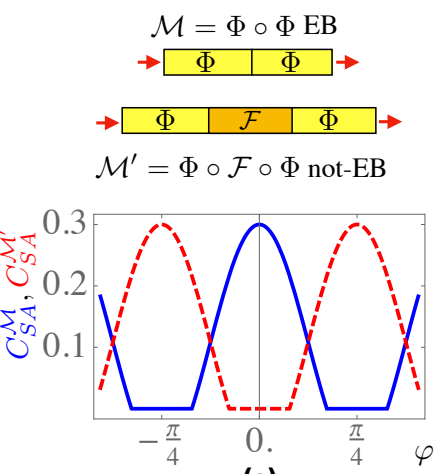

(a)

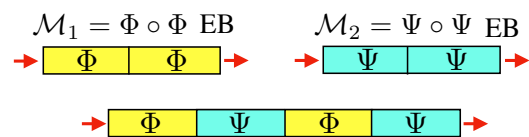

$\mathcal{M}^{\prime}=\Psi \circ \Phi \circ \Psi \circ \Phi$ not-EB

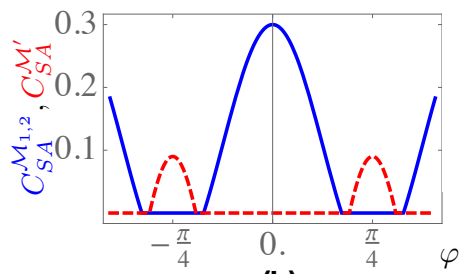

(b)
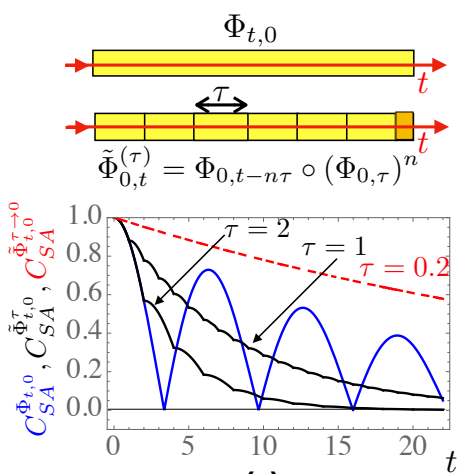

(c)

Figure 2. Techniques for recovering the transmission of quantum correlations in presence of an EB dynamics. The curves refer to concurrence values of Choi-Jamiołkowski state for the indicated maps. (a) The blue solid line corresponding to $\mathcal{M}=\Phi \circ \Phi$ with $\Phi=\mathcal{A}_{\eta=0.3} \circ \mathcal{U}_{\varphi}$, shows that $\mathcal{M}$ is EB around $\varphi= \pm \pi / 4$, which can be "corrected" thanks to a filter $\mathcal{F}=\mathcal{U}_{\pi / 4}$ as showed by the red dashed curve. (b) The blue solid line represents the value of concurrence yielding the same value for the channels $\mathcal{M}_{1}=\Phi \circ \Phi$ and $\mathcal{M}_{2}=\Psi \circ \Psi$ with $\Phi=\mathcal{A}_{\eta=0.3} \circ \mathcal{U}_{\varphi}$ and $\Psi=\mathcal{U}_{\varphi} \circ \mathcal{A}_{\eta=0.3}$. Two EB-regions emerge, located around $\pm \pi / 4$. The red dashed map, referring to the composite map $\mathcal{M}^{\prime}=\Psi \circ \Phi \circ \Psi \circ \Phi=\left(\mathcal{A}_{\eta=0.3} \circ \mathcal{U}_{\varphi}\right) \circ\left(\mathcal{A}_{\eta=0.3} \circ \mathcal{U}_{\pi / 4}\right) \circ\left(\mathcal{A}_{\eta=0.3} \circ \mathcal{U}_{\varphi}\right) \circ\left(\mathcal{A}_{\eta=0.3} \circ \mathcal{U}_{\pi / 4}\right)$, shows that in such regions correlations can be transmitted again. (c) Concurrence values of the Choi-Jamiołkowski state of a non-Markovian evolution for a quibt $S$ interacting with a bosonic bath with spectral density $J(\omega)=\frac{1}{2 \pi} \frac{\alpha \ell^{2}}{\left(\omega_{0}-\omega\right)^{2}+\ell^{2}}$ with $\alpha \geq 0$ the effective coupling constant, $\ell$ the width of the Lorentzian spectrum, and the frequency $\omega_{0}$ gauging the energy gap of $S$. Here we have set $\alpha=5 \omega_{0} t$ and $\ell=0.1 \omega_{0}$. The blue solid line refers to the associated map $\Phi_{t, 0}$, while the solid black lines and the red dashed one refer to the perturbed mapping $\tilde{\Phi}_{t}^{(\tau)}$ for smaller and smaller values of $\tau$.

Acknowledgments: A.D.P. is deeply grateful to V. Giovannetti, A. Mari, P. Mataloni, F. Sciarrino, S. Maniscalco, A. Porzio, P. Verrucchi and A. Cuccoli for fundamental discussions leading to a fruitful collaboration on the topic addressed in this manuscript. Moreover, A.D.P. acknowledges the financial support from the University of Florence in the framework of the University Strategic Project Program 2015 (Project No. BRS00215).

Conflicts of Interest: The author declares no conflict of interest.

\section{References}

1. Holevo, A.S. Quantum Systems, Channels, Information: A Mathematical Introduction; De Gruyter: Berlin, Germany, 2012.

2. De Pasquale, A.; Giovannetti, V. Quantifying the noise of a quantum channel by noise addition. Phys. Rev. A 2012, 86, 052302.

3. Holevo, A.S. Quantum coding theorems. Russ. Math. Surv. 1999, 53, 1295-1331.

4. Bellomo, B.; De Pasquale, A.; Gualdi, G.; Marzolino, U. A tomographic approach to non-Markovian master equations. J. Phys. A Math. Theor. 2010, 43, 395303.

5. Gatto, D.; De Pasquale, A.; Giovannetti, V. Degradation of entanglement in Markovian noise. Phys. Rev A 2019, 99, 032307.

6. Gorini, V. Kossakowski, A. and Sudarshan, E. C. G., J. Math. Phys. 1976, 17, 821.

7. Lindblad G., Commun. Math. Phys. 1976, 48, 119.

8. Rivas, A.; Huelga, S.F.; Plenio, M.B. Entanglement and non-Markovianity of quantum evolutions. Phys. Rev. Lett. 2010, 105, 050403.

9. Cuevas, Á.; De Pasquale, A.; Mari, A.; Orieux, A.; Duranti, S.; Massaro, M.; Di Carli, A.; Roccia, E.; Ferraz, J.; Sciarrino, F.; et al. Cut-and-paste restoration of entanglement transmission. Phys. Rev. A 2017, 96, 012314.

10. Cuevas, Á.; De Pasquale, A.; Mari, A.; Orieux, A.; Duranti, S.; Massaro, M.; Di Carli, A.; Roccia, E.; Ferraz, J.; Sciarrino, F.; et al. Amending entanglement-breaking channels via intermediate unitary operations. Phys. Rev. A 2017, 96, 022322. 
11. De Pasquale, A.; Mari, A.; Porzio, A.; Giovannetti, V. Amendable Gaussian channels: Restoring entanglement via a unitary filter. Phys. Rev. A 2013 87, 062307

12. Rigovacca, L.; Farace, A.; De Pasquale, A.; Giovannetti, V. Gaussian Discriminating Strength. Phys. Rev. A 2015, 92, 042331.

13. Bullock, T.; Cosco, F.; Haddara, M.; Raja, S.H.; Kerppo, O.; Leppäjärvi, L.; Siltanen, O.; Talarico, N.W.; De Pasquale, A.; Giovannetti, V.; et al. Entanglement Protection via Periodic Environment Resetting in Continuous Time Quantum Dynamical Processes. Phys. Rev. A 2018, 98, 042301.

14. Facchi, P.; Pascazio, S. Progress in Optics; Wolf, E., Ed.; Elsevier: Amsterdam, The Netherlands, 2001; Volume 42, Chapter 3, pp. 147-218.

(C) 2019 by the authors. Licensee MDPI, Basel, Switzerland. This article is an open access article distributed under the terms and conditions of the Creative Commons Attribution (CC BY) license (http://creativecommons.org/licenses/by/4.0/). 